\title{
Acknowledgment to Reviewers of Biomimetics in 2020
}

\section{Biomimetics Editorial Office}

MDPI AG, St. Alban-Anlage 66, 4052 Basel, Switzerland

Peer review is the driving force of journal development, and reviewers are gatekeepers who ensure that Biomimetics maintains its standards for the high quality of its published papers. Thanks to the cooperation of our reviewers, in 2020, the median time to first decision was 21.5 days and the median time to publication was 53.5 days. The editors would like to express their sincere gratitude to the following reviewers for their precious time and dedication, regardless of whether the papers were finally published:

Abella, Laura Sanchez

Adnan, Memic

Ahmadi, Seyed F.

Alfredo, Gonzalez-Perez

Ali, Jamel

Al-Obaidi, Karam

Al-Radhi, Mohammed Salah

Alwattar, Tahseen A.

Anne-Claire, Groo

Antoniadis, Aristomenis

Astruc-Diaz, Fanny

Atrian-Blasco, Elena

Attik, Nina G.

Babaei, Mahnoush

Bae, Haejin

Balayeva, Narmina O.

Barath, Zoltan

Barbieri, Filippo

Barrientos, Antonio

Bastrzyk, Anna

Berthold-Pluta, Anna

Bertl, Adam

Bhounsule, Pranav A.

Bohinc, Klemen

Bonilla, Clara Eugenia Plazas

Bonser, Richard

Bottari, Fabio

Campisi, Giuseppina

Canfield, Stephen

Carmagnola, Irene

Cataldi, Amelia

Cerbu, Camelia

Chowdhury, Md Raihan

Chu, Chun Hung

Clegg, John R.

Copot, Cosmin
Costantino, Marco Fabio

Cui, Haitao

D'Aurelio, Roberta

Dahms, Hans-Uwe

D'Angelo, Ivana

David, Bierbach

Davidova, Marie

Degli Esposti, Lorenzo

Den Toonder, Jaap

Deschner, James

Ding, Xi-Lun

Diogo, Patrícia

Do, Thanh N.

Dong, Guoying

Dong, Mingdong

Ehlicke, Franziska

Ene, Razvan

Estevez, Alberto T.

Farzaneh, Helena $\mathrm{H}$.

Ferreira, Barbara

Fraga-García, Paula

Francolini, Iolanda

$\mathrm{Fu}$, Chao

Fuest, Matthias

Gadomska-Gajadhur, Agnieszka

Gangadharan, Rajan

Gao, Yun

Gebeshuber, Ille

Ghidelli, Matteo

Ghodake, Gajanan

Giberti, Hermes

Gomes, Ana P.

Gradov, Oleg V.

Hasling, Karen M.

Hayes, Samantha

Herbig, Maik 
Hesselberg, Thomas

Ho, Chia-che

Horner, Andreas

Hosseini Nejad, Elham

Iconaru, Simona L.

Jain, Anuj

Jeronimidis, George

Ji, Ai-hong

Karcz, Dariusz

Khassawna, Thaqif E.

Khetani, Salman R.

Kim, Young-Rok

Kohsaka, Ryo

Korupalli, Chiranjeevi

Krater, Martin

Krieger, Axel

Kucinska, Malgorzata

Kumar, Harish

Lamas, María Isabel

Lauder, George

Lee, Jong Yong

Lee, Junsoo

Leech, Damien

Liu, Yuxin

Lopez-Cara, Luisa Carlota

Lord, Nathan P.

Magdysyuk, Oxana V.

Magyari, Klara

Manini, Paola

Mansoori, Ali

Marciello, Marzia

Martin-Gomez, Cesar

Mayama, Hiroyuki

Mazza, Edoardo

McHenry, Matthew

Mead, Taryn

Mishra, Alaknanda

Miyaji, Akimitsu

Mlynarczyk, Dariusz

Mondal, Sayan

Monteiro, Carlos

Mueller, Jochen

Muntean, Alexandrina

Nakielski, Pawel

Naumowicz, Monika

Navabpour, Parnia

Niclos Gutierrez, Juan

Niewiarowski, Peter

Noskov, Boris A.

Nurunnabi, Mohammad

Ohya, Yoshikazu
Patane, Luca

Patnaik, Sourav S.

Perez-Ramirez, Carlos

Pitucha, Monika

Prado Da Fonseca, Vinicius

Qian, Jin-yuan

Ramachandran, Roshini

Ramos, Laurence

Real Oliveira, Maria E. C.D.

Rey, Juan

Robert, Newcomb

Robertson, James

Rovalo, Erin

Rusu, Laura Cristina

Saeki, Daisuke

Samal, Sangram

Samuel, Shani

Santarelli, Andrea

Scribante, Andrea

Shach-Pinsly, Dalit

Shin, SangJoon

Simanjuntak, Firman Mangasa

Singh, Anirudha

Skaba, Dariusz

Slegers, Nathan J.

Sodergard, Anders

Sotomayor, Maria

Suarato, Giulia

Swaileh, Wassim

Tampieri, Anna

Tanase, Constantin

Tartaglia, Gianluca

Tawil, Bill J.

Tenkumo, Taichi

Thakar, Dhruv

Thakur, Vijay

Thiria, Benjamin

Toby, Brian

Touchie, Marianne

Touloupaki, Eleftheria

Tsuji, Hideto

Tursi, Antonio

Tytell, Eric

Van De Kamp, Thomas

Vanaga, Ruta

Verstegen, Monique M. A.

Vikraman, Dhanasekaran

Visser, Arnoud

Vladkova, Todorka

Wandelt, Klaus

Wang, Bing 
Pastor, JESUS

Westhauser, Fabian

Wie, Jeong Jae

Williams, John

Wu, Chia-Wen

Yilmaz, Mehmet

$\mathrm{Yu}, \mathrm{Lu}$
Weissburg, Marc

Yuan, Haobo

Zhang, Sung-Uk

Zhao, Jie

Zhu, Hanxing

Zubareva, Ekaterina V.

Zwart, Hub 